\title{
Rede de apoio social da família para o cuidado da criança com paralisia cerebral
}

\author{
Social support network of the family for the care of children with cerebral palsy
}

Red de apoyo social de la familia para el cuidado del niño con parálisis cerebral

\author{
Giovana Calcagno Gomes', Bianca Contreira de Jung", Camila Magroski Goulart Nobre ${ }^{\prime \prime \prime}$, \\ Pâmela Kath de Oliveira Nörberg ${ }^{\prime v}$, Carolina Domingues Hirsch ${ }^{v}$, Fabiele Dias Dresch ${ }^{\text {VI }}$
}

\begin{abstract}
RESUMO
Objetivo: analisar a rede de apoio social utilizada pela família para cuidar a criança com paralisia cerebral. Método: pesquisa qualitativa, descritiva, realizada com 12 mães de crianças com paralisia cerebral atendidas em unidade de pediatria do Hospital Universitário de Rio Grande. Os dados foram coletados nos meses de agosto e setembro de 2018, por meio de entrevista semiestruturada, e submetidos à análise temática. Resultados: identificou-se a rede de apoio social formada pelos familiares, amigos, vizinhos, profissionais de saúde e ancorada pela fé em Deus. As mães foram protagonistas do cuidado e algumas não receberam nenhum tipo de apoio para os cuidados dos filhos. Conclusão: a rede de apoio social contribuiu para a superação de dificuldades da família e para o cuidado da criança, sendo fonte de apoio emocional, financeiro, ajuda com o transporte, remédios e orientação dos profissionais de saúde/enfermagem.
\end{abstract}

Descritores: Família; paralisia cerebral; criança; apoio social.

\section{ABSTRACT}

Objective: to know the social support network used by the family to care for children with cerebral palsy. Method: this qualitative and descriptive research was conducted with twelve mothers of children with cerebral palsy assisted at a pediatric unit of a University Hospital in Rio Grande. Data were collected in August and September 2018 through semi-structured interviews and submitted to thematic analysis. Results: we identified the social support network consisting of family, friends, neighbors, health professionals, and faith in God. Mothers were protagonists of care and some received no support for childcare. Conclusion: the social support network contributed to overcome family difficulties and childcare, being a source of emotional, financial support, help with transportation, medicine, and guidance from health/nursing professionals.

Descriptors: Family; cerebral palsy; child; social support.

\section{RESUMEN}

Objetivo: conocer la red de apoyo social utilizada por la familia para cuidar a niños con parálisis cerebral. Método: investigación cualitativa, descriptiva, realizada con doce madres de niños con parálisis cerebral atendidas en una unidad de pediatría de un Hospital Universitario de Río Grande. Los datos se recopilaron en agosto y septiembre de 2018 a través de entrevistas semiestructuradas y se sometieron a análisis temáticos. Resultados: identificamos que la red de apoyo social estaba formada por familiares, amigos, vecinos, profesionales de la salud y fe en Dios. Las madres fueron protagonistas de la atención y algunas no recibieron apoyo para el cuidado de los niños. Conclusión: la red de apoyo social contribuyó a superar las dificultades familiares y el cuidado de los niños, siendo una fuente de apoyo emocional y financiero, ayuda con el transporte, medicamentos y orientación de profesionales de la salud/enfermeros.

Descriptores: Familia; parálisis cerebral; niño; apoyo social.

\section{INTRODUÇÃO}

A paralisia cerebral (PC) ou encefalopatia crônica não progressiva caracteriza-se por uma condição psicomotora especial decorrente de lesão do sistema nervoso central, ainda em fase de maturação, manifestando-se na primeira infância, comumente antes dos 18 meses de idade. Tal condição influencia o contexto de vida familiar, pois exige a necessidade de cuidados especiais à criança e é permeada de dúvidas, medos, conflitos e incertezas ${ }^{1,2}$.

Os dados epidemiológicos apontam prevalência variável em países desenvolvidos de 1,5 a 2,5 por 1000 nascidos vivos com PC. No Brasil, estima-se o surgimento aproximado de 30.000 a 40.000 novos casos por ano. Frente a essa situação, as famílias necessitam se reorganizar e buscar formas efetivas de cuidado $3^{4}$.

Para as famílias e crianças com necessidades especiais, a rede de apoio social significa amparo para enfrentar a nova condição de vida apresentada. A rede social diz respeito aos aspectos relacionados ao indivíduo, de modo estrutural ou institucional, suas diversas relações e vínculos sociais, com recursos de apoio, podendo incluir igrejas e instituições de saúde ${ }^{5}$.

'Enfermeira. Doutora. Professor Associado III. Universidade Federal do Rio Grande. Brasil. E-mail: giovanacalcagno@furg.br

"Enfermeira. Mestre, Universidade Católica de Pelotas. Universidade Federal do Rio Grande. Brasil. E-mail: biancajung@ucpel.edu.br

'"'Enfermeira. Mestre. Universidade Federal do Rio Grande. Brasil. E-mail: kamy_magroski@yahoo.com.br

IVEnfermeira. Doutora, Universidade Federal do Rio Grande. Brasil. E-mail: pamelakathpko@yahoo.com.br

vEnfermeira. Doutora, Universidade Federal do Rio Grande. Brasil. E-mail: hirsch.carolina@gmail.com

v'Acadêmica de Enfermagem, Universidade Federal do Rio Grande. Brasil. E-mail: fabieleddresch@gmail.com 
Por rede de apoio compreende-se o processo de interação entre pessoas ou grupos que estabelecem vínculos de amizade e de informação, recebe-se apoio material, emocional, afetivo, contribuindo para o bem-estar recíproco, construindo fatores positivos na prevenção e manutenção da saúde ${ }^{5}$. Diante da demanda de cuidados exigidos apresentada pelas crianças com PC, é necessário o envolvimento de uma rede de apoio social, integrada pelos serviços e equipes de saúde/enfermagem, familiares e comunidade, capaz de auxiliar os cuidadores na tomada de decisões, transformando-os em sujeitos autônomos. Assim, a pesquisa objetivou analisar a rede de apoio social utilizada pela família para cuidar da criança com PC.

\section{REVISÃO DE LITERATURA}

A PC é uma doença crônica incapacitante com diversos graus de dependência, que impõe a necessidade constante de cuidados pela família, além de ser a causa mais comum de deficiência física grave que acomete crianças ${ }^{2}$. Caracterizase por desordens do tônus, da postura e do movimento e problemas musculoesqueléticos secundários. É uma condição heterogênea, com múltiplas causas, vários padrões de neuropatologia nas imagens cerebrais, diversos tipos clínicos e patologias do desenvolvimento associadas, tais como autismo, deficiência mental, alterações perceptivas, de comunicação e de comportamento, epilepsia e deficiência visual $\left.\right|^{6,7}$.

Estudo que objetivou analisar a satisfação de cuidadores com os apoios sociais disponíveis a crianças com PC identificou alta satisfação com o apoio recebido e indicou o nível de satisfação geral alto nos pais de crianças com maior comprometimento, por meio do apoio material, afetivo, emocional, informação e interação social positiva ${ }^{8}$.

Pesquisa identificou que nem sempre a rede é fortalecida e capaz de oferecer o apoio necessário ao enfrentamento adequado da doença. No entanto, em alguns momentos da vida a criança recebeu diferentes tipos de apoio oferecidos por vínculos fortalecidos. Assim, ressalta-se que os profissionais da saúde precisam direcionar seu olhar para a criança, ouvindo-a em suas singularidades, ajudando a identificar elos em sua rede social que possam Ihe fornecer o apoio de que precisam para enfrentar a doença crônica ${ }^{9}$.

Estudo ressaltou que mães de crianças relataram a importância do apoio recebido para a superação de adversidades e readaptação aos cuidados da criança. Os dados demonstraram várias fontes de estresse e escassez de recursos, assim como a influência da falta de apoio na saúde física e emocional das participantes ${ }^{10}$.

A rede social no cuidado à criança com doença crônica favoreceu o compartilhamento de informações e experiências, momentos de descontração e auxílio na aquisição de insumos para o tratamento, com repercussão positiva no contexto familiar ${ }^{11}$. As mulheres, em geral, são as principais responsáveis por cuidar dos filhos e, com isso, são expostas a altas exigências e sobrecarga.

\section{METOdOLOGIA}

Estudo qualitativo do tipo descritivo, realizado na unidade de pediatria de um hospital universitário, localizado do município de Rio Grande, Rio Grande do Sul/Brasil. Participaram da pesquisa 12 cuidadores de crianças com diagnóstico de PC que atenderam aos seguintes critérios de inclusão: ser o principal familiar cuidador da criança, ter 18 anos ou mais e prestar-Ihe cuidados diretos em casa. Foram excluídos cuidadores que, mesmo estando presentes durante a hospitalização da criança, promoviam seu cuidado eventualmente.

Os cuidadores foram contatados na própria unidade de pediatria, sendo convidados a participar do estudo. FoiIhes explicado o objetivo, metodologia e método de coleta dos dados. A coleta de dados foi iniciada posteriormente à assinatura do Termo de Consentimento Livre e Esclarecido pelos participantes. Os dados foram coletados entre agosto e setembro de 2018 , em ambiente anexo à unidade de pediatria, com a utilização de entrevistas semiestruturadas, que tiveram tempo médio de duração de 40 minutos. Foram questionados acerca do apoio social recebido para o cuidado da criança.

Os dados foram tratados pelo processo de análise de conteúdo ${ }^{12}$, a qual se dividiu em três etapas: na pré-análise, realizou-se a leitura flutuante e a formulação de hipóteses; a segunda etapa constituiu-se na exploração do material com a codificação dos dados e classificação em categorias; e, a última compreendeu o tratamento dos resultados obtidos, inferência, interpretação e reflexão. Discutiram-se a categoria Rede de apoio social e as subcategorias: Mães protagonistas do cuidado; Apoio de familiares; Apoio de amigos e vizinhos; Apoio de profissionais de saúde; e Apoio na fé em Deus, que emergiram da análise de conteúdo dos depoimentos.

Foram respeitados os princípios éticos da pesquisa envolvendo seres humanos, conforme a Resolução nํ466/12. $\mathrm{O}$ anonimato das participantes foi garantido e suas falas identificadas pela letra $\mathrm{F}$ (Familiar), seguida do número correspondente à entrevista. O projeto de pesquisa foi aprovado pelo Comitê de Ética em Pesquisa na Área da Saúde, tendo recebido o parecer favorável no 197/2018 e CAAE 94590218.7.0000.5324. 


\section{RESULTADOS E DISCUSSÃO}

Participaram do estudo 12 familiares cuidadores, sendo 10 mães da criança com PC, um pai e uma cuidadora institucional. Suas idades variaram entre 23 anos e 53 anos. Oito possuem Ensino Fundamental incompleto, duas Ensino Médio incompleto e duas Ensino Médio completo. Em relação à renda familiar, uma não soube informar, uma recebe meio salário mínimo, quatro - um salário mínimo, uma cerca de $\mathrm{R} \$ 1.400,00$, três cerca de $\mathrm{R} \$ 1.800,00$, uma cerca de $\mathrm{R} \$ 2.000,00$ e outra cerca de $\mathrm{R} \$ 2.500,00$.

Os conteúdos das entrevistas com os familiares cuidadores foram englobados na categoria rede de apoio social e desmembrados em cinco subcategorias, que são tratadas a seguir.

\section{Mães protagonistas do cuidado}

As mães foram protagonistas do cuidado integral da criança com PC pela importância que os filhos assumem em suas vidas. Entretanto, algumas mencionaram não ter uma rede de apoio social, tendo como impacto a sobrecarga física e emocional.

Como é que eu vou te explicar, família entre aspas, porque eu assumi a bronca toda sozinha. (F5)

Praticamente eu me viro um pouco só, porque ele [o pai] também tem as tarefas dele. (F9)

Ninguém, só eu cuido dela. (F8)

Agora mesmo estava precisando de alguém para ficar com ele. Ninguém se ofereceu. É difícil (F4)

O pai dele não ajuda em nada, nem se oferece. Poderia muito bem me ajudar, dizer assim: Vou pagar uma pessoa por fora para te dar uma força. Eu saio e minhas irmãs ficam em cinco minutos me ligando: Tu vais demorar muito? (F5)

As mães foram as protagonistas do cuidado da criança com PC, dedicando-se de forma integral, assumindo as demandas determinadas pelas necessidades dos seus filhos. Estudo apontou a qualidade de vida significativamente insatisfatória da mãe cuidadora de criança com PC, que apresentou mais fragilidade física e emocional ${ }^{13}$.

Os dados deste estudo demonstraram a falta da rede de apoio social de certas mães que assumiram exclusivamente o cuidado de seus filhos, sem receber ajuda de pessoas próximas. Pesquisa apresentou, como facilitadores do apoio da família, os amigos e a sociedade para a realização de atividades e melhoria das condições de vida da criança e família ${ }^{14}$.

As mães mencionaram as sobrecargas emocional e física ocasionadas pelos cuidados contínuos conferidos à criança. Pesquisa com objetivo de apreender as facilidades e as dificuldades da família no cuidado às crianças com PC revelou que as dificuldades são mais evidentes comparadas às facilidades e trazem repercussões nas atividades de vida diárias como alimentação, banho e locomoção, pois as crianças ficam a cada dia mais dependentes dos cuidadores ${ }^{9}$.

\section{Apoio de familiares}

As mães mencionaram o apoio de familiares na rotina de cuidados da criança. Houve ajuda nos cuidados em geral e dos irmãos menores, nas trocas com a mãe durante a internação hospitalar, auxílio financeiro, para o transporte e medicamentos.

Somente da minha filha tenho ajuda. Sou eu e ela e, às vezes, da minha mãe. Somos as três que encaramos, dia e noite, no caso 24 horas, mas mais somente eu, direto com ele (F5).

Me auxiliam nos cuidados que precisamos, como medicação e com tudo que ele precise. (F3)

O pai largou o emprego para poder me ajudar. (F6)

Enquanto ele está aqui no hospital, elas vêm para a gente pode ir em casa tomar um banho, cuidar dos outros filhos. (F1)

Sempre tem alguém na volta para ajudar. Se eu preciso de carro ou preciso de dinheiro. (F2)

A rede de apoio social é necessária para enfrentar a complexidade imposta pelas necessidades constantes em todas as dimensões de cuidado da criança. Os achados de estudo corroboram a importância da rede de suporte formada pela família, que ofereceu apoio social, emocional, guia cognitivo, ajuda material, atenuando as limitações impostas pela deficiência ${ }^{15}$.

A maioria das mães mencionou a existência do apoio familiar como uma fortaleza para o enfrentamento das circunstâncias impostas pela condição da criança. Pesquisa apontou dados semelhantes, evidenciando que o núcleo familiar contribui para o fortalecimento das dimensões emocional, instrumental, informacional e $\operatorname{cog}^{2}$ itiva $^{16}$.

Estudo demonstrou a importância do apoio do marido e demais membros da família para a adaptação e reorganização da vida. São recursos usados pelas mulheres para facilitar o desempenho do papel materno, enfrentar desafios e minimizar o estresse ${ }^{10}$. 


\section{Apoio de amigos e vizinhos}

Neste estudo, a rede de apoio social configurou-se para além da família, o apoio dos amigos e vizinhos foi essencial para enfrentar as dificuldades. O apoio envolveu a dimensão de ofertar ajuda para o transporte da criança, aquisição de remédios e, ainda, significou o suporte emocional.

Recebi apoio da minha vizinha e patroa, são ótimas pessoas e da Neneca. (F3)

Os amigos se aproximaram muito mais. Eu sei que se eu ligar, se precisar de qualquer coisa, eu sempre tenho quem venha para me ajudar. Às vezes, a dificuldade é com o remédio que eu tenho de comprar, mas tudo que eu preciso eu sempre tenho. Às vezes, quando a situação está difícil com a saúde dele mesmo, sempre tem um abraço. (F7)

E os amigos que também têm carro, às vezes que eu preciso, eu ligo quando o meu marido não pode ir e peço: Tu podes me levar amanhã de manhã a tal lugar? (F2)

Estudo demonstrou que as famílias possuem rede de apoio restrita e para a demanda de cuidados contam apenas com o apoio do marido, avós, filhos e padrinhos ${ }^{5}$. Já na presente pesquisa as mães que apontaram a existência da rede de apoio social mencionaram o apoio de amigos e vizinhos.

Pesquisas apontaram a rede social formada pelos amigos como um recurso essencial de apoio para a família a fim de ajudá-la nos momentos difíceis ${ }^{4,16}$. Neste estudo, as mães buscaram o apoio necessário nos amigos e vizinhos para a superação dos obstáculos.

\section{Apoio de profissionais de saúde}

Para as mães, os profissionais de saúde/enfermagem foram importantes fontes de apoio emocional e físico, pois elas se sentiram mais tranquilas e conseguiram descansar. Também receberam as informações adequadas sobre os cuidados com a criança e esclarecimento as dúvidas.

Estão sempre explicando. Ontem, eu até perguntei por que ele estava babando, porque ele nunca tinha colocado tanta baba e elas disseram: é normal mãe. Não te preocupas. Tranquilizam. (F1)

Os médicos sempre foram prestativos comigo, sempre acompanhando ele. Eles tratam o melhor que podem. (F4)

As enfermeiras fazem tudo e explicam, mas eu esqueço. Quando eu estou no hospital eu não faço nada. Descanso. Eu só fico olhando elas fazerem. (F8)

A parte de aspiração, da gastrostomia, isso tudo é o cuidado das enfermeiras. Então, enquanto ele está no hospital a gente dispõe dessa ajuda porque as enfermeiras estão auxiliando. (F6)

Tudo que eu sei para cuidar dele eu aprendi aqui, dentro do hospital. Recebo orientação da enfermeira de como é que tu fazes isso, como é que faz aquilo. (F7)

A atuação dos profissionais de saúde/enfermagem no processo de tratamento dessas crianças foi relevante por proporcionar um cuidado integral à criança e família. Nesse sentido, reconhece-se a importância de uma assistência profissional ampliada que possa lidar não apenas com a criança com PC, mas com intervenções junto aos familiares, garantindo-lhes bem-estar, qualidade de vida e apoio contínuo ${ }^{9,17}$.

Neste estudo, os dados apontaram a relevância positiva do apoio profissional/enfermagem na melhoria da qualidade de vida das mães, do bem-estar psicossocial. Assim, os profissionais contribuem para diminuição do estresse e para amortecer a sobrecarga de cuidado, pois fortalecem a rede de apoio social, com a corresponsabolização e empoderamento da família para o cuidado da criança ${ }^{17}$.

Destarte, os profissionais de saúde/enfermagem foram essenciais para amparar a família nos momentos de dificuldades, transmitindo-lhe segurança e tranquilidade, além de possibilitar o conhecimento científico sobre o cuidado. Desse modo, o comportamento e as informações fornecidas pelos profissionais contribuem para a compreensão do processo de adoecimento e enfrentamento da condição da criança ${ }^{16}$.

\section{Apoio na fé em Deus}

Outra importante fonte de apoio familiar é a fé em Deus. Esta fé dá forças e esperança à família para lutar pela criança e acreditar na sua recuperação, com a possibilidade de se desenvolver e adquirir melhor qualidade de vida.

Graças a Deus, com a graça de Deus, ela está aí. (F9)

A gente vê a batalha dela todo dia. A gente luta porque ela vai ficar boa, vai com certeza. A gente tem fé em Deus que ela vai melhorar. (F3)

Eu rezo para Deus todos os dias para ver ele melhorar, ter uma qualidade de vida melhor. Deus está me dando força e eu estou aguentando. (F5) 
Tudo o que eu preciso, graças a Deus, consigo, tudo que ele precisou até agora eu sempre consegui. Eu comecei a leva-lo à Igreja, tenho muita fé e ele começou a se desenvolver. (F4)

A crença e a fé foram determinantes para as mães se recomporem e buscarem encarar a condição dos filhos. Dessa forma, a fé/espiritualidade aparece como suporte emocional, que influencia na aceitação, adaptação e consolo da família em relação à doença, assim como na segurança e capacidade de vivenciar essa situação ${ }^{16}$.

Estudo salientou que as famílias buscam a dimensão espiritual para o acalento diante das adversidades encontradas, elas veem na crença/fé um sustentáculo para o enfrentamento diário da condição crônica de seus filhos ${ }^{18}$. A família, muitas vezes, é impulsionada para a busca de respostas, ajuda e/ou significados em outras dimensões da vida, que não só a material, a cognitiva e a afetiva, chegando à espiritual.

A nova rotina imposta aos familiares pela condição da criança com PC, permeada por dificuldades cotidianas, faz com que as famílias procurem na crença/fé fortalezas para o enfrentamento dos obstáculos. Pesquisa demonstrou que os pais invocaram Deus para superar sentimentos e pensamentos negativos sobre os seus filhos e enfrentar o futuro nebuloso diante da PC, pois a espiritualidade, na maioria das vezes, passa a ser um suporte para aceitação da cronicidade da condição de vida da criança ${ }^{19}$.

Embora as dificuldades permeiem o contexto das famílias, elas foram capazes de encontrar apoio e auxílio na rede de apoio social, tal rede se entrelaça para que as necessidades dos familiares cuidadores e da criança sejam atendidas da melhor maneira possível ${ }^{17}$.

\section{CONCLUSÃO}

Ao pesquisar sobre a rede de apoio social dos cuidadores de crianças com PC em unidade de pediatria hospitalar do município de Rio Grande, mães perceberam-se como protagonistas do cuidado conferido aos filhos e, também, pelas dificuldades que algumas delas tiveram pela falta de apoio.

De modo geral, a rede de apoio foi ampliada e fortalecida, sendo formada pelos amigos, vizinhos, profissionais de saúde e ancorada na fé em Deus. Identificaram-se, por meio dos resultados, que essas formas de apoio significaram ter ajuda das pessoas do convívio para os cuidados da criança, orientações e esclarecimentos por parte dos profissionais de saúde/enfermagem. A fé em Deus apresenta-se como baluarte para a superação de dificuldades da família.

Conclui-se, que apesar haver rede de apoio social para o cuidado à criança, a mãe apresenta sobrecargas emocional e física. Destaca-se que as famílias passaram por mudanças que levaram à readaptação de suas rotinas, precisando enfrentar diariamente desafios e dificuldades que circundaram os cuidados com a criança.

Aponta-se, como limitação da pesquisa, a restrição do campo de estudo em somente uma unidade de pediatria, pois apesar de sua relevância, não possibilita generalizar os resultados para outros municípios, por constituir uma amostra local não representativa do universo. Entretanto, a pesquisa trata de uma realidade mundial, servindo de subsídio para novas investigações sobre o tema.

\section{REFERÊNCIAS}

1. Graham HK, Rosenbaum P, Paneth N, Dan B, Lin JP, Damiano DL, et al. Cerebral palsy. Nat Rev Dis. Primers [internet]. 2016 [cited 2019 Oct 28]; 2(15082):1-25. DOI: https://doi.org/10.1038/nrdp.2015.82

2. Santos BAS, Milbrath VM, Freitag VL, Nunes NJS, Gabatz RIB, Silva MS. The impact of cerebral palsy diagnosis from the perspective of the family. REME rev. min. Enferm. [internet]. 2019 [cited 2019 Oct 28]; 23:e-1187. Available from: http://www.reme.org.br/artigo/detalhes/1330

3. Caneco EDOV, Milbrath VM, Freitag VL, Amestoy SC. Revealing the diagnosis of severe perinatal asphyxia to the family: a perspective of health professionals. Arq. ciênc. saúde. [internet]. 2016 [cited 2019 Oct 15]; 23(2):23-9. DOI: https://doi.org/10.17696/2318-3691.23.2.2016.310

4. Rocha MC, Carvalho MS, Fossa AM, Pedroso GE, Rossato LM. Necessities and Difficulties of the Family that Live the Experience of to Have a Child With Hydrocephalus. Rev. bras. educ. espec. [internet]. 2015 [cited 2019 Oct 15]; 15(40):49-66. DOI: http://dx.doi.org/10.15600/2238-1244/sr.v15n40p49-66

5. Barbosa TA, Reis KMN, Lomba GO, Alves GV, Braga PP. Support network and social support for children with special health care need. Rev Rene (Online). [internet]. 2016 [cited 2019 Oct 28]; 17(1):60-6. DOI: https://doi.org/10.15253/21756783.2016000100009

6. MacLennan AH, Thompson SC, Gecz J. Cerebral palsy: causes, pathways, and the role of genetic variants. Am. j. obstet. gynecol. [internet]. 2015 [cited 2019 Oct 28]; 213(6):779-88. DOI: https://doi.org/10.1016/j.ajog.2015.05.034

7. Pfeifer LI, Silva DB, Lopes PB, Matsukura TS, Santos JL, Pinto MP. Social support provided to caregivers of children with cerebral palsy. Child care health dev. [internet]. 2014 [cited 2019 Oct 15]; 40 (3):363- 9. DOI: http://dx.doi.org/10.1111/cch.12077

8. Maltoni J, Lisboa CSM, Matos MG, Teodoro MLM, Neufeld CB. Cultural adaptation of the Health Behaviour Protocol in Schoolaged Children for the Brazilian reality. Psicol. teor. prát. [internet]. 2018 [cited 2019 Oct 28]; 20(2):161-174. Available from: http://pepsic.bvsalud.org/scielo.php?script=sci_arttext\&pid=S1516-36872019000300003 
9. Silva MEA, Moura FM, Albuquerque TM, Reichert APS, Collet N. Network and social support in children with chronic diseases: understanding the child's perception. Texto \& contexto enferm. [internet]. 2017 [cited 2019 Oct 28]; 26(1):1-10. DOI: http://dx.doi.org/10.1590/0104-07072017006980015

10. Ribeiro MFM, Vandenberghe L, Prudente COM, Vila VSC, Porto CC. Cerebral Palsy: how the child's age and severity of impairment affect the mother's stress and coping strategies. Ciênc. saúde coletiva (Online) [internet]. 2016 [cited 2019 Oct 15]; 21(10):3203-12. DOI: http://dx.doi.org/10.1590/1413-812320152110.17352016

11. Pennafort VPS, Queiroz MVO, Nascimento LC, Guedes MVC. Network and social support in family care of children with diabetes. Rev. bras. enferm. (Online) [internet]. 2016 [cited 2019 Oct 29]; 69(5):856-63. DOI: http://dx.doi.org/10.1590/0034-7167-20150085

12. Bardin L. Análise de conteúdo. 3ạ reimp. Lisboa (Pt): Edições.70; 2011.

13. Wu J, Zhang J, Hong Y. Quality of life of primary caregivers of children with cerebral palsy: a comparison between mother and grandmother caregivers in Anhui province of China. Child care health dev. [internet]. 2017 [cited 2019 Oct 29]; 43(5):718-24. DOI: https://doi.org/10.1111/cch.12464

14. Earde PT, Praipruk A, Rodpradit P, Seanjumla P. Facilitators and Barriers to Performing Activities and Participation in Children with Cerebral Palsy: Caregivers' Perspective. Pediatr. phys. ther. [internet]. 2018 [cited 2019 Oct 29]; 30(1):27-32. DOI: http://dx.doi.org/10.1097/PEP.0000000000000459

15. Brignol P, Shoeller SD, Silva, DMG, Lopes SGR, Souza SS. Support network for persons with physical disabilities. Rev. enferm. UERJ. [internet]. 2017 [cited 2019 Oct 29]; 25:e18758. DOI: https://doi.org/10.12957/reuerj.2017.18758

16. Polita NB, Tacla MT. Network and social support to families of children with cerebral palsy. Esc. Anna Nery Rev. Enferm. [internet]. 2014 [cited 2019 Oct 29]; 18(1):75-81. Available from: http://eean.edu.br/detalhe_artigo.asp?id=1001

17. Dantas MAS, Nóbrega VM, Fechine CPNS, IMB Torquato, Assis WD, Collet N. Professional care for children with cerebral palsy and their families. Rev. enferm. UERJ. [internet]. 2017 [cited 2019 Oct 29]; 25:e18331. DOI: http://dx.doi.org/10.12957/reuerj.2017.18331

18. Dezoti AP, Alexandre AM, Freire MH, Mercês NN, Mazza VA. Social support to the families of children with cerebral palsy. Acta Paul. Enferm. (Online). 2015 [cited 2019 Oct 29]; 28(2):172-6. DOI: http://dx.doi.org/10.1590/1982-0194201500029

19. Nimbalkar S, Raithatha S, Shah R, Panchal DA. A qualitative study of psychosocial problems among parents of children with cerebral palsy attending two tertiary care hospitals in western India. ISRN Family Med. [internet]. 2014 [cited 2019 Oct 29]; 769619: 1-6. DOI: http://dx.doi.org/10.1155/2014/769619 